\title{
Correction to: 1 Versus 2-cm Excision Margins for pT2-pT4 Primary Cutaneous Melanoma (MelMarT): A Feasibility Study
}

\author{
Marc D. Moncrieff, MD FRCS(Plast.) ${ }^{1}$, David Gyorki, FRACS ${ }^{2}$, Robyn Saw, FRACS ${ }^{3}$, Andrew J. Spillane, \\ FRACS $^{3}$, John F. Thompson, MD, FRACS, FACS ${ }^{3}$, Howard Peach, FRCS(Plast. $)^{4}$, Deemesh Oudit, FRCS(Plast. $)^{5}$, \\ Jenny Geh, FRCS(Plast.) ${ }^{6}$, Peter Dziewulsk, FRCS(Plast.) ${ }^{7}$, Ewan Wilson, FRCS(Plast. ${ }^{8}$, Paolo Matteucci, \\ FRCS(Plast.) ${ }^{9}$, Rowan Pritchard-Jones, FRCS(Plast. ${ }^{10}$, Roger Olofsson Bagge, MD, PhD ${ }^{11}$, Frances C. Wright, \\ MD, M.Ed ${ }^{12}$, Nic Crampton, FRACS ${ }^{13}$, Oliver Cassell, FRCS(Plast. $)^{14}$, Navid Jallali, FRCS(Plast. $)^{15}$, Adam Berger, \\ MD $^{16}$, John Kelly, MD FACD ${ }^{17}$, Stephen Hamilton, FRCS(Plast. $)^{18}$, Amer Durrani, FRCS(Plast. $)^{19}$, Serigne Lo, \\ $\mathrm{PhD}^{3,20}$, Elizabeth Paton, $\mathrm{MSc}^{3,20}$, and Michael A. Henderson, FRACS ${ }^{2}$ \\ ${ }^{1}$ Norfolk \& Norwich University Hospital, Norwich, UK; ${ }^{2}$ Peter MacCallum Cancer Centre, Melbourne, Australia; \\ ${ }^{3}$ Melanoma Institute Australia, Sydney, Australia; ${ }^{4}$ Leeds Teaching Hospitals, Leeds, UK; ${ }^{5}$ Christie NHS Trust, \\ Manchester, UK; ${ }^{6}$ Guy's \& St Thomas's NHS Trust, London, UK; ${ }^{7}$ St Andrew's Centre for Burns \& Plastic Surgery, \\ Chelmsford, UK; ${ }^{8}$ North Bristol NHS Trust, Bristol, UK; ${ }^{9}$ Hull \& East Yorkshire NHS Trust, Hull, UK; ${ }^{10}$ Mersey Centre \\ for Burns \& Plastic Surgery, Liverpool, UK; ${ }^{11}$ Sahlgrenska University Hospital, Göteborg, Sweden; ${ }^{12}$ Sunnybrook Health \\ Sciences Centre, Toronto, Canada; ${ }^{13}$ Gold Coast Melanoma Clinic, Queensland, Australia; ${ }^{14}$ Oxford University Hospitals \\ NHS Trust, Oxford, UK; ${ }^{15}$ Imperial Hospital NHS Trust, London, UK; ${ }^{16}$ Jefferson University Hospitals, Philadelphia; \\ ${ }^{17}$ The Alfred Hospital, Melbourne, Australia; ${ }^{18}$ Royal Free Hospital NHS Trust, London, UK; ${ }^{19}$ Cambridge University \\ Hospitals, Cambridge, UK; ${ }^{20}$ Australia \& New Zealand Melanoma Trials Group, North Sydney, Australia
}

\section{CORRECTION TO: ANN SURG ONCOL HTTPS://DOI.ORG/10.1245/S10434-018-6470-1}

In the original article John F. Thompson was inadvertently omitted as an author. The author list is updated as shown in this correction.

The original article can be found online at https://doi.org/10.1245/ s10434-018-6470-1. 